\title{
Total Skin-Sparing Mastectomy
}

National Cancer Institute

\section{Source}

National Cancer Institute. Total Skin-Sparing Mastectomy. NCI Thesaurus. Code C106320.

Mastectomy with preservation of the breast skin and the skin of the nipple and areola. 